IMS

médecine/sciences $1993 ; 9: 9-11$

\title{
LA BIOLOGIE MOLÉCULAIRE DES RÉCEPTEURS ET L'ESSOR D'UNE NOUVELLE PHARMACOLOGIE
}

\section{Jean-Charles Schwartz}

\section{RÉFÉRENCES}

1. Black JW, Duncan WAM, Durant CJ, Gancllin CR, Parsons ME. Definition and antagonism of histamine $\mathrm{H}_{2}$ receptors. Nature $1972 ; 236: 385-90$.

2. Arrang JM, Garbarg M, Schwartz JC Autoinhibition of brain histamine release mediated by a novel class $\left(\mathrm{H}_{3}\right)$ of histamine receptor. Nature $1983 ; 302: 832-7$.

3. Arrang JM, Garbarg M, Lancelot JC, et al. Highly potent and sclective ligands for histaminc $\mathrm{H}_{3}$ receptors. Nature $1987 ; 327$ : 117-23.

4. Changcux JP. The acetylcholine receptor: an "allostcric " membrane protein. Harvey Lect $1981 ; 75: 85-254$.

5. Lefkowitz RJ, Kobilcka BK, Caron MG. The new biology of drug receptors. Biochem Pharmacol 1989 ; 38 : 2941-8.

6. Kuntz ID. Structure-bascd strategies for drug design and discovery. Science 1992 ; 257: 1078-82.

\footnotetext{
ADRESSE

J.-C. Schwartz : professeur à l'université RenéDescartes et à l'Institut universitaire de France, directeur de l'unité de neurobiologie et pharmacologie. Inscrm U. 109, centrc Paul-Broca, 2 ter, rue d'Alćsia, 75014 Paris, France.
}

es récepteurs des neurotransmetteurs et hormones sont aussi, potentiellement, des récepteurs de médicaments. Ce truisme souligne comment l'évolution rapide de nos connaissances dans le domaine des récepteurs, dont témoignent plusieurs articles de ce numéro, est de nature à entraîner une évolution corrélative de la pharmacologie, conçue comme l'art de mettre au point des médicaments nouveaux. Dans les deux domaines de recherche, en effet, c'est l'utilisation des moyens et des résultats de la biologie moléculaire qui constitue le moteur de l'évolution.

Pendant longtemps, l'étude des effets de médicaments potentiels a été largement indirecte, l'interaction entre ceux-ci et leur cible moléculaire étant analysée en quantifiant sur animal entier, organe ou tissu isolé soit une réponse, soit, plus récemment, la compétition avec une sonde radioactive plus ou moins spécifique. Dans le meilleur des cas, un "modèle" théorique approximatif du site de reconnaissance du récepteur était déduit des relations progressivement établies entre la structure chimique de nombreux ligands et leur activité biologique. Ces modèles ont, certes, montré leur intérêt opérationnel (notamment en suggérant la synthèse de nouveaux ligands mieux reconnus) mais aussi leurs limites, le processus de mise au point de molécules actives nouvelles n'étant jamais devenu entièrement rationnel comme celui qui pourrait découler de la connais- sance de la structure réelle des cibles visées.

Or, le clonage moléculaire d'un nombre croissant de récepteurs est en train de modifier cette pharmacologie classique.

La pharmacologie classique : $d u$ médicament au récepteur et du récepteur au gène

Depuis Ehrlich jusqu'aux années soixante-dix, le récepteur était demeuré une entité nécessaire mais essentiellement théorique selon l'adage Corpora non agunt nisi fixata*. $\mathrm{Au}$ cours de cette période, la découverte d'un nouveau récepteur reposait sur le choix d'une réponse biologique appropriée et sur la mise au point d'agents chimiques, effecteurs ou antagonistes sélectifs. Ainsi les suggestions de récepteurs multiples de la noradrénaline et de l'histamine, faites par Ahlquist et Schild respectivement, n'ont pu aboutir à l'identification pharmacologique formelle des récepteurs $\beta$-adrénergiques et $\mathrm{H}_{2}$ de l'histamine qu'après plusieurs années. La mise au point d'antagonistes sélectifs, dans les deux cas précédents, par sir James Black et ses collègues, est en effet le résultat d'un patient travail de synthèse et d'essai de plusieurs centaines de molécules [1]. Plus récemment, et pour prendre un exemple de notre laboratoire, il nous fallait encore quatre années d'un travail d'investigation de même type

* Les substances n'agissent que si elles sont fixées. 
pour confirmer [2] l'existence, que nous avions précédemment suggérée [3], du troisième récepteur $\left(\mathrm{H}_{3}\right)$ de l'histamine.

Ce sont encore des ligands chimiques, sous forme de sondes réversibles ou irréversibles ou encore de réactifs pour chromatographie d'affinité, qui ont été les instruments clés pour l'isolement des premiers récepteurs [4,5]. De là a découlé la détermination d'une partie de leur séquence ouvrant la voie au clonage de leur gène.

Un tel processus est inévitablement lent car il comporte deux étapes difficiles à franchir : la mise au point de ligands sélectifs et l'isolement de protéines très peu abondantes.

Or le processus de clonage de gènes de récepteurs s'est profondément modifié au cours des dernières années. Cela devrait avoir des conséquences profondes sur la mise au point de nouvelles classes de médicaments $[6,7]$.

La "pharmacologie inverse": du gène au récepteur et $d u$ récepteur au médicament

Le clonage moléculaire des premiers récepteurs a révélé leur appartenance à de très larges " superfamilles " dont les membres présentent, cntre eux, d'utiles homologies de séquences. C'est principalement l'utilisation de ces homologies qui a permis le clonage rapide des gènes de nouveaux récepteurs au moyen des techniques d'hybridation croisée ou d'amplification itérative (PCR). L'expression de ces récepteurs en cellules cucaryotes transfectées a alors révélé que plusieurs d'entre eux étaient "pharmacologiquement inconnus". Cela est bien illustré par des exemples pris dans lcs familles de récepteurs adrénergiques dopaminergiques (voir l'article de P. Sokoloff et al., p. 12 de ce numéro) ou sérotoninergiques (voir l'article de M. Hamon et H. Gozlan, p. 21 de ce numéro) qui, tous, appartiennent à la superfamille des récepteurs à sept domaines transmembranaires couplés à une protéine $G$ [8]. $\mathrm{Au}$ sein de cette superfamille, le nombre de membres clonés et identifiés ne cesse de croître, mais nombreux aussi sont, dans plusieurs laboratoires, les "récepteurs orphelins" ou " récepteurs en quête d'une fonc- tion ", c'cst-à-dire dont le ligand endogène - neurotransmetteur, hormone, ou facteur de croissance reste à identifier.

Dans la superfamille des récepteurscanaux qui comprend notamment les récepteurs nicotiniques et GABAergiques, la multiplicité est non moins fascinante qui, cette fois, résulte de la combinaison de sous-unités variées (voir l'article de N. Le Novère et al., p. 41 de ce numéro).

Dans ces divers cas, la découverte inattendue d'un grand nombre de cibles moléculaires nouvelles constitue à la fois une chance et un défi pour les pharmacochimistes.

- La chance réside dans la possibilité qui leur est offerte de développer de manière rationnelle de nouvelles classes d'agents thérapeutiques, particulièrement dans des domaines tels que ceux de la pharmacologie du système nerveux central ou du tractus gastro-intestinal, où les progrès ont été rares au cours des dernières années. En effet, les nombreux "sous-types" de récepteurs que dévoile la biologie moléculaire diffèrent entre eux non seulement par leur séquence mais aussi par leur pharmacologic, leur(s) systèmc(s) de signalisation intracellulairc et leur expression tissulairc. Dans la plupart des cas, l'arsenal thérapeutique préexistant ne comporte que des agents ne permettant pas de les différencier complètement. Un bon exemple est fourni par les neurolcptiques, antagonistes de la dopamine, bloquant simultanément plusieurs sous-types de récepteurs, dont un seul pourrait sous-tendre leur efficacité antipsychotique, le blocage des autres étant responsable des nombreux effets secondaires que produiscnt ces médicaments et qui limitent considérablement leur usage (voir l'article de P. Sokoloff et al., p. 12 de ce numéro). En revanche, on peut raisonnablement attendre d'unc mise au point d'agents nouveaux, présentant une sélectivité pharmacologique élevée, un spectre d'activité plus pur, lié à l'expression discrète de chacun des sous-types de récepteurs dans des populations neuronales distinctes. Ainsi, dans de nombreux cas, le rêve, longtemps poursuivi en vain par les pharmacologues, d'agents présentant une sélectivité tissulaire, voire cellulaire, élevée ne paraît plus hors de portée. Par exemple, l'expression très contrastée des cinq sous-typcs de récepteurs muscariniques (et de nombreux sous-types de récepteurs nicotiniques) par des populations cellulaires distinctes dans le cerveau et les tissus périphériques, semble de naturc à renouvcler le spectre des applications thérapeutiques de ces vieux médicaments que sont les agents cholinergiques ou anticholinergiques : lc clonage des récepteurs muscariniques nous a révélé leur manque de sélectivité, qui limite considérablement certains de leurs emplois.

- Le défi lancé aux pharmacochimistes par l'existence de ces cibles multiples - cst lui aussi - très clair : développer des ligands susceptibles de différencier des récepteurs parfois très homologues ou pour lesquels la pharmacologie est encore quasi inexistante. Cepcndant, la biologic moléculaire vient apporter un certain nombre d'atouts pour relever ce défi.

Les récepteurs recombinants : vers une mise au point rationnelle de nouveaux médicaments à partir de leurs cibles

En dépit de revendications, très souvent usurpées, de "mise au point rationnelle " de médicaments, le travail du pharmacochimiste est largement resté celui d' " un tireur d'élite visant, dans l'obscurité, une cible mal délimitée ". En effet, c'est encore le plus souvent au prix d'un patient cffort de synthèse de nombreux analogues chimiques, dont les effets biologiques sont alors analysés sur des systèmes plus ou moins complexes, que sont sélectionnées, plus que véritablement conçues, les nouvelles molécules à visée thérapeutique.

Il n'est pas exagéré de dire que la biologie moléculaire est en train de bouleverser ce processus de mise au point d'une manière double : d'unc part en facilitant l'essai biologique des médicaments synthétisés et, d'autre part, en dévoilant enfin la structure moléculaire des cibles que ccs médicaments sont destinés à atteindre.

En effet, les lignćes de cellules aisément cultivables, comme des fibroblastes ou même des bactéries, et 
exprimant à lcur surfacc une haute densité d'un sous-type de réceptcur, après transfection du gènc correspondant sous contrôlc d'un promoteur viral, constituent des outils particulièrement performants pour l'cssai initial de ligands potentiels. Dans certains cas, celui des récepteurs muscariniques par exemple, ces modèles sont les seuls utilisables, car il est souvent difficile d'isoler un tissu exprimant naturellement un seul sous-type de ces récepteurs. Dans tous les cas, le haut niveau d'expression (généralement dix à cent fois supérieur à celui des tissus les plus riches) constitue un avantage déterminant cn termes de précision des résultats. La possibilité d'évalucr de manière simple l'action des médicaments sur un récepteur humain que l'on a fait exprimer par une de ces cellules constitue aussi un avantage déterminant dans les cas où des différences pharmacologiqucs liées à l'espècc existent, comme cela a été démontré pour lc réceptcur $\mathrm{H}_{1}$ dc l'histamine [9] ou certains sous-types des récepteurs de la sérotonine (voir l'article de M. Hamon et H. Gozlan, p. 21 de ce numéro).

Ces lignées de ccllules nc facilitent pas seulement les essais de candidats médicaments par radioliaison mais aussi ceux qui reposent sur la mesure d'unc réponsc biologique telle que taux d'un second messager ou flux ionique. La grande amplitude de cettc réponsc, résultant de la fortc densité de réccpteurs exprimés, permet d'évaluer avec unc précision accruc une propriété essentielle des agonistes, leur " activité intrinsèque ", c'est-à-dire leur plus ou moins grandc capacité d'induire une transconformation productive du réccpteur. Ces modèles cellulaircs permettent de n'avoir recours que tardivement à l'essai sur animal entier, lequel porte alors sur des molécules déjà présélectionnées in vitro. En outre, le recours à des animaux transgéniques devrait êtrc de nature à améliorcr des essais in vivo, particulièrement dans la mesurc où des anomalics qualitatives ou quantitatives de réccpteurs vicnnent à être identifiées. On peut raisonnablement espérer que de telles approches conduiront cnfin à la misc au point de modèles animaux réalis-

$\mathrm{m} / \mathrm{s} n^{\circ} 1$ vol. 9, janvier 93 tes d'affections neuropsychiatriques humaines, modèles dont lc défaut a largement compromis l'essor de la psychopharmacologie au cours des dernières déccnnies.

Mais plus importante encore pour le développement de nouvclles stratégies de mise au point de médicaments est la connaissance de l'anatomie fonctionnelle de leur cible, la molécule de protéine réceptrice. Ainsi, la seule séquence en acides aminés bientôt complétée par les données de la mutagenèse dirigće et celles du marquage irréversible de la protéine par ses ligands, rapprochées de la séquence de protéines homologues ayant pu être soumiscs à des analyses physiques de structure (comme cela cst le cas pour la bactériorhodopsine) cst en train de fournir, avec de plus en plus de détails, une image des sites de reconnaissance des ligands au sein des récepteurs. Cette image, quoique encorc largement conjecturale, est affinée par la mise en œuvre de modélisations moléculaires de la protéine, particulièrement celles d'entre elles qui tiennent comptc des données de la mutagenèse dirigée (voir l'article de $M$. Hibert et al., p. 31 de ce numéro).

Une étape déterminantc sera franchie lorsque l'interaction des ligands avec les groupes fonctionnels du site de reconnaissance pourra être analyscee en trois dimensions ct, si possible, en temps réel au moyen de méthodes physiques approprićcs telles que microscopie électroniquc, diffraction des électrons ou des rayons $\mathrm{X}$ et résonance magnétique nucléaire ( $\mathrm{RMN}$ ). C'est cette information qui, convenablement exploitéc par la modélisation moléculaire, devrait conduire à la misc au point réellement rationnclle de médicaments. Une des conditions préalables, la disponibilité de quantités suffisantes de protéine, est en principe satisfaite par l'utilisation de récepteurs recombinants, synthétisables en quantité illimitéc. En revanchc, certains problèmes restent non résolus : ils résident dans la cristallisation de ces protéines transmembranaircs et dans l'analyse de protéines d'une taille encore supérieure aux limites qu'autorise la RMN à ce jour, en dépit de progrès récents spectaculaires [10].
La mise au point d'une nouvelle classc de médicaments était restéc un événement rare. On pcut prophétiser sans grand risquc quc, après un ccrtain délai lić aux phases incompressibles d'études toxicologiques et cliniques, les prochaines déccnnies vont connaître un enrichisscment considérable de notre arscnal thérapeutique

\section{RÉFÉRENCES}

7. Schwartz JC. Molecular biology of drug receptors and the advent of reverse pharmacology. In : Wermuth CG, Kof ar N, König $\mathrm{H}$, Metcalf W, cds. Medicinal Chemistry for the XXIst Century. Oxford : Blackwcll Scientific Publications, $1992: 63-71$.

8. Savarcse TM, Frascr CM. In vitro mutagencsis and the scarch for structure-function relationships among $G$ protcin-coupled reccptors. Biochem J $1992 ; 283$ : 1-19.

9. Schwartz JC, Arrang JM, Garbarg M, Pollard H, Ruat M. Histamincrgic transmission in the mammalian brain. Physiol Rev $1991 ; 71: 1-51$

10. Clore GM, Gronenborn AM. Structures of larger proteins in solution : threc- and four-dimensional heteronuclear NMR spectroscopy. Science 1992; 292 : 1390-9.

\section{TIRÉS A PART}

J.-C. Schwartz. 